\title{
Universal Ratios and Correlation Functions
}

\section{G. Delfino and G. Mussardo*}

Scuola Internazionale Superiore di Studi Avanzati (SISSA)

INFN, Sezione di Trieste (Italy)

\begin{abstract}
We review some recent results concerning the quantitative analysis of the universality classes of two-dimensional statistical models near their critical point. We also discuss the exact calculation of the two-point correlation functions of disorder operators in a free theory of complex bosonic and fermionic field, correlators ruled by a Painlevè differential equation.
\end{abstract}

\section{Introduction}

One of the most important successes of Quantum Field Theory (QFT) in recent years is the quantitative analysis of the universality classes of two-dimensional statistical mechanical models near their second order phase transition points. This has been possible, in particular, thanks to new developments in the methods of computing off-critical correlation functions. The aim of this talk is to review some of the recent results relative to these two important subjects. Our discussion is divided in two parts: in the first part, we shall see how correlation functions can be used to extract useful numbers (the so-called universal ratios) that can be directly compared with experiments. In the second part, we shall concentrate on a particularly interesting class of models, those of fermionic and bosonic theories with a $Z_{n}$ symmetry, for which there is an elegant approach to derive differential equations satisfied by the two-point functions of their disorder operators.

\section{Universal Amplitude Ratios}

Consider a statistical model with $n$ relevant fields $\varphi_{i}(x)$ at criticality. Near the critical point, its action can be parameterised as

$$
\mathcal{A}=\mathcal{A}_{C F T}+g_{i} \int \varphi_{i}(x) d^{D} x
$$

where $\mathcal{A}_{C F T}$ is the action which encodes the data of the Conformal Field Theory of the critical point. The short-distance behaviour of the two-point functions is given by

$$
\left\langle\varphi_{i}(x) \varphi_{i}(0)\right\rangle \simeq \frac{1}{|x|^{4 \Delta_{i}}}
$$

\footnotetext{
${ }^{*}$ Speaker.
} 
for $|x| \rightarrow 0$, where $\Delta_{i}$ is the conformal dimension of the operator $\varphi_{i}$. Hence, their conjugate coupling constants $g_{i}$ behave as $g_{i} \sim \Lambda^{D-2 \Delta_{i}}$, where $\Lambda$ is a mass scale. Away from criticality there will be generally a finite correlation length $\xi$ which in the thermodynamical limit scales as $\xi \sim a\left(K_{i} g_{i}\right)^{-\frac{1}{D-2 \Delta_{i}}}$, where $a \sim \Lambda^{-1}$ may be regarded as a microscopic length scale. The dimensionless quantities $K_{i}$ are non-universal metric factors specific of the system representing the universality class and depending on the units chosen for measuring the external sources $g_{i}$. If there are several deformations of the conformal action, the most general expression for the scaling form of the correlation length may be written as

$$
\xi=\xi_{i} \equiv a\left(K_{i} g_{i}\right)^{-\frac{1}{D-2 \Delta_{i}}} \mathcal{L}_{i}\left(\frac{K_{j} g_{j}}{\left(K_{i} g_{i}\right)^{\phi_{j i}}}\right)
$$

where $\phi_{j i} \equiv \frac{D-2 \Delta_{j}}{D-2 \Delta_{i}}$ are the so-called crossover exponents whereas $\mathcal{L}_{i}$ are universal homogeneous scaling functions of the ratios $\frac{K_{j} g_{j}}{\left(K_{i} g_{i}\right)^{\phi_{j i}}}$. There are of course several (but equivalent) ways of expressing these scaling forms, depending on which coupling constant is selected as a prefactor. In the limit where $g_{l} \rightarrow 0(l \neq i)$ but $g_{i} \neq 0$, equation $(\overline{2}, \overline{2} .2 i)$ becomes

$$
\xi_{i}=a \xi_{i}^{0} g_{i}^{-\frac{1}{D-2 \Delta_{i}}} \quad, \quad \xi_{i}^{0} \sim K_{i}^{-\frac{1}{D-2 \Delta_{i}}} .
$$

Let us consider now the free-energy $\hat{f}\left[g_{1}, \ldots, g_{n}\right]$, a dimensionless quantity defined by

$$
e^{-\hat{f}\left(g_{1}, \ldots, g_{n}\right)}=\int \mathcal{D} \phi e^{-\left[\mathcal{A}_{C F T}+\sum_{i=1}^{n} g_{i} \int \varphi_{i}(x) d^{D} x\right]} .
$$

Under the hypothesis of hyperscaling, its singular part (per unit of volume) $f\left[g_{1}, \ldots, g_{n}\right]$ is proportional, in the thermodynamical limit, to the $D$-th power of the correlation length. Depending on the scaling form adopted for the correlation length, we have correspondingly several (but equivalent) ways of parameterizing this quantity

$$
f_{i}\left[g_{1}, \ldots, g_{n}\right] \equiv\left(K_{i} g_{i}\right)^{\frac{D}{D-2 \Delta_{i}}} \mathcal{F}_{i}\left(\frac{K_{j} g_{j}}{\left(K_{i} g_{i}\right)^{\phi_{j i}}}\right)
$$

The functions $\mathcal{F}_{i}$ are universal homogeneous scaling functions of the ratios $\frac{K_{j} g_{j}}{\left(K_{i} g_{i}\right)^{\phi_{j i}}}$.

From the above expression we can derive the expectation values of several quantities. We will use the notation $\langle\ldots\rangle_{i}$ to denote expectation values computed in the off-critical theory obtained by keeping (at the end) only the coupling constant $g_{i}$ different from zero. Basic quantities are the vacuum expectation values (VEV) of the fields $\varphi_{j}$ which can be parameterized as

$$
\left\langle\varphi_{j}\right\rangle_{i}=-\left.\frac{\partial f_{i}}{\partial g_{j}}\right|_{g_{l}=0} \equiv B_{j i} g_{i}^{\frac{2 \Delta_{j}}{D-2 \Delta_{i}}},
$$

with

$$
B_{j i} \sim K_{j} K_{i}^{\frac{2 \Delta_{j}}{D-2 \Delta_{i}}}
$$

The above relations can be equivalently expressed as

$$
g_{i}=D_{i j}\left(\left\langle\varphi_{j}\right\rangle_{i}\right)^{\frac{D-2 \Delta_{i}}{2 \Delta_{j}}}
$$


with

$$
D_{i j} \sim \frac{1}{K_{i} K_{j}^{\frac{D-2 \Delta_{i}}{2 \Delta_{j}}}} .
$$

The generalized susceptibilities of the model are defined by

$$
\hat{\Gamma}_{j k}^{i}=\frac{\partial}{\partial g_{k}}\left\langle\varphi_{j}\right\rangle_{i}=-\left.\frac{\partial^{2} f_{i}}{\partial g_{k} \partial g_{j}}\right|_{g_{l}=0} .
$$

They are obviously symmetrical in the two lower indices. By extracting their dependence on the coupling constant $g_{i}$, they can be expressed as

$$
\hat{\Gamma}_{j k}^{i}=\Gamma_{j k}^{i} g_{i}^{\frac{2 \Delta_{j}+2 \Delta_{k}-D}{D-2 \Delta_{i}}}
$$

with

$$
\Gamma_{j k}^{i} \sim K_{j} K_{k} K_{i}^{\frac{2 \Delta_{j}+2 \Delta_{k}-D}{D-2 \Delta_{i}}}
$$

The various quantities obtained by taking the derivatives of the free-energy obviously contain the quantities $K_{i}$ which make their values not universal. However, it is easy and always possible to consider special combinations thereof in such a way to cancel out all metric factors. Universal Ratios usually considered in the literature are those given below

$$
\begin{gathered}
\left(R_{c}\right)_{j k}^{i}=\frac{\Gamma_{i i}^{i} \Gamma_{j k}^{i}}{B_{j i} B_{k i}} ; \\
\left(R_{\chi}\right)_{j}^{i}=\Gamma_{j j}^{i} D_{j j} B_{j i}^{\frac{D-4 \Delta_{j}}{2 \Delta_{j}}} ; \\
R_{\xi}^{i}=\left(\Gamma_{i i}^{i}\right)^{1 / D} \xi_{i}^{0} ; \\
\left(R_{A}\right)_{j}^{i}=\Gamma_{j j}^{i} D_{i i}^{\frac{4 \Delta_{j}+2 \Delta_{i}-2 D}{D-2 \Delta_{i}}} B_{i j}^{\frac{2 \Delta_{j}-D}{\Delta_{i}}} ; \\
\left(Q_{2}\right)_{j k}^{i}=\frac{\Gamma_{j j}^{i}}{\Gamma_{j j}^{k}}\left(\frac{\xi_{k}^{0}}{\xi_{j}^{0}}\right)^{D-4 \Delta_{j}} ;
\end{gathered}
$$

From their definition, these quantities are pure numbers attached to the universality classes and therefore they can be used to characterize them. Contrary to the critical exponents (which are characteristic of the critical point) they carry information about the scaling region. Moreover, the amplitude ratios are numbers which typically present significant variations between different classes of universality, whereas the critical exponents usually assume small values which only vary by a small percent when changing the universality class. Hence the universal ratios may be ideal marks of the critical scaling regime [i] experimental point of view, it should be simpler to measure universal amplitude ratios rather than critical exponents: to determine the former quantities one needs to perform several measurements at a single, fixed value of the coupling which drives the system away from criticality whereas to determine the latter, one needs to make measurements over several decades along the axes of the off-critical couplings. Moreover, although not all of them are independent, the universal ratios are a larger set of numbers than the critical exponents and therefore permit a more precise determination of the class of universality. 


\section{Quantum Field Theory Approach}

It is interesting to see how in two dimensions Quantum Field Theory provides a powerful approach to compute the Universal Ratios of a given class of universality in a non-perturbative way. In the following we concentrate mostly our attention on the off-critical models obtained as deformations of the so-called Minimal Models of two-dimensional Conformal Field Theory [를,

As we have seen before, each coupling constant $g_{i}$ relative to the relevant operator $\varphi_{i}(x)$ of the model under investigation is a dimensional quantity which can be related to the lowest mass-gap $m_{i}=\xi_{i}^{-1}$ of the off-critical theory according to the formula

$$
m_{i}=\mathcal{C}_{i} g_{i}^{\frac{1}{2-2 \Delta_{i}}}
$$

When the QFT associated to the action $(\overline{2} 2 . \overline{1})$ is integrable, the pure number $\mathcal{C}_{i}$ can be exactly determined by means of the Thermodynamical Bethe Ansatz [i $\left[\begin{array}{l}\overline{1} \\ \mathbf{1}\end{array}\right.$ integrable, the constant $\mathcal{C}_{i}$ can be nevertheless determined by a numerical method, based on

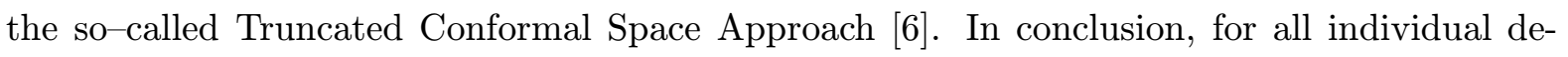
formations of a given model, we are able to completely set the relationship which links the coupling constant to the mass-gap of the theory and therefore to switch freely between these two variables.

Additional quantities which can be determined by QFT are the matrix elements of the order parameters, the simplest ones being the vacuum expectation values (VEV). They are parameterised as

$$
\left\langle\varphi_{j}\right\rangle_{i}=B_{j i} g_{i}^{\frac{\Delta_{j}}{1-\Delta_{i}}}
$$

When the theory is integrable, the constant $B_{j i}$ can be fixed exactly, thanks to the results of a remarkable series of papers $\left[\begin{array}{l}\bar{T}_{1}, \\ 1,8\end{array}\right]$. When it is not integrable, the constant $B_{j i}$ can be nevertheless estimated by means of a numerical approach, as firstly shown in $[\underline{9} \overline{1}]$. Hence, we are able to determine completely also these quantities. Moreover, as shown in [1] of the numerical approach of ref. [igin often leads to a reasonable estimate of the matrix elements of the order parameters between the vacuum states and some of the excited states, as for instance $\left\langle 0\left|\varphi_{j}\right| A_{k}\right\rangle_{i}$ where $A_{k}$ is a one-particle state of mass $M_{k}$. These quantities turn out to be useful for obtaining sensible approximation of the large-distance behavior of several correlators.

Another important and useful piece of information on the off-critical models can be obtained by exploiting the properties of the stress-energy tensor $T_{\mu \nu}(x)$. In the presence of the perturbing field $\varphi_{i}$, the trace of the stress-energy tensor is given by

$$
\Theta(x)=2 \pi g_{i}\left(2-2 \Delta_{i}\right) \varphi_{i} .
$$

It enters a useful sum rule, the so called $\Delta$-theorem [i] $\left.{ }_{2}^{1}\right]$

$$
\Delta_{j}=-\frac{1}{4 \pi\left\langle\varphi_{j}\right\rangle_{i}} \int d^{2} x\left\langle\Theta(x) \varphi_{j}(0)\right\rangle_{i}^{c}
$$

which relates the conformal dimension $\Delta_{j}$ of the field $\varphi_{j}$ to its VEV and to the integral of its connected off-critical correlator with $\Theta(x)$. It is easy to see that the above formula simply expresses the content of the fluctuation-dissipation theorem and when the above integral 
diverges, so does the VEV in the denominator, in such a way that eq. ( $(\overline{3} \cdot \overline{3}-\overline{4})$ always keeps its

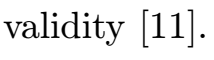

As discussed above, basic quantities entering the universal ratios are the generalized susceptibilities $\Gamma_{j k}^{i}$. By using the fluctuation-dissipation theorem, they are given by

$$
\hat{\Gamma}_{j k}^{i}=\int d^{2} x\left\langle\varphi_{j}(x) \varphi_{k}(0)\right\rangle_{i}^{c}
$$

By extracting their dependence on the coupling constant $g_{i}$, we have $\hat{\Gamma}_{j k}^{i}=\Gamma_{j k}^{i} g_{i}^{\frac{\Delta_{j}+\Delta_{k}-1}{1-\Delta_{i}}}$ with

$$
\Gamma_{j k}^{i}=\mathcal{C}_{i}^{2 \Delta_{j}+2 \Delta_{k}-2} \int d \tau \frac{1}{\tau^{2 \Delta_{j}+2 \Delta_{k}}} Q_{j k}(\tau)
$$

Some of the above susceptibilities can be determined exactly, such as the components $\Gamma_{i k}^{i}$, whose values are provided by the $\Delta$-theorem sum rule

$$
\Gamma_{i k}^{i}=-\frac{\Delta_{k}}{1-\Delta_{k}} B_{k i}
$$

In all other cases, when an exact formula is not available, the strategy to evaluate the generalized susceptibilities relies on two different possible representations of the correlators. As we will see, these representations have the advantage of converging very rapidly in two distinct regions and to smoothly join in between.

The first representation is based on Conformal Perturbation Theory (with the use of the non-analytic expression of the VEV) [1] $\left.{ }_{1}^{1} \overline{3}\right]$. In this approach the two-point correlators are expressed as

$$
\left\langle\varphi_{i}(x) \varphi_{j}(0)\right\rangle=\sum_{p} C_{i j}^{p}(g ; x)\left\langle A_{p}(0)\right\rangle
$$

where the structure functions $C_{j k}^{p}(g ; x)$ admit the expansion

$$
C_{i j}^{p}(g ; x)=r^{2\left(\Delta_{p}-\Delta_{i}-\Delta_{j}\right)} \sum_{n=0}^{\infty} C_{i, j}^{p(n)}\left(g r^{2-2 \Delta_{\Phi}}\right)^{n},
$$

$(r=|x|)$ and can be computed perturbatively in $g$, the coupling conjugated to the perturbing operator $\varphi$. For instance, the first order correction to the conformal structure constant $C_{i, j}^{p(0)}$ is determined by [1] $\underline{1} \overline{3}$ ]

$$
C_{i, j}^{p(1)}=-\int^{\prime} d^{2} w\left\langle A^{p}(\infty) \varphi(w) \varphi_{i}(1) \varphi_{j}(0)\right\rangle_{C F T}
$$

where the prime indicates a suitable infrared (large distance) regularization of the integral. This representation allows a very efficient estimate of the correlation function in its short distance regime $r \ll \xi$.

The second representation is based on the Form Factors (which will be discussed in more detail below) and allows an efficient control of the large distance behavior, i.e. when $r \gg \xi$. In this second representation, one makes use of the knowledge of the off-critical mass spectrum of the theory to express the correlators as

$$
\left\langle\varphi_{i}(x) \varphi_{j}(0)\right\rangle=\sum_{n=0}^{\infty} g_{i, j}^{(n)}(r)
$$


where

$$
\begin{aligned}
& g_{i, j}^{(n)}(r)=\int_{\theta_{1}>\theta_{2} \ldots>\theta_{n}} \frac{d \theta_{1}}{2 \pi} \ldots \frac{d \theta_{n}}{2 \pi} e^{-r \sum_{k=1}^{n} m_{k} \cosh \theta_{k}} \\
& \times\left\langle 0\left|\varphi_{i}(0)\right| \ldots A_{a_{n}}\left(\theta_{n}\right)\right\rangle\left\langle\ldots A_{a_{n}}\left(\theta_{n}\right)\left|\varphi_{j}(0)\right| 0\right\rangle .
\end{aligned}
$$

$\left|A_{a_{1}}\left(\theta_{1}\right) \ldots A_{a_{n}}\left(\theta_{n}\right)\right\rangle$ are the multi-particle states relative to the excitations of mass $m_{k}$, with relativistic dispersion relations given by $E=m_{k} \cosh \theta, p=m_{k} \sinh \theta$, where $\theta$ is the rapidity variable. The spectral representation (3.12i) is an expansion in the parameter $e^{-\frac{r}{\xi}}$, where $\xi^{-1}=m_{1}$ is the lowest mass-gap.

Basic quantities of the large distance approach are the Form Factors (FF), i.e. the matrix elements of the operators $\varphi_{i}$ on the physical asymptotic states

$$
F_{a_{1}, \ldots, a_{n}}^{\varphi_{i}}\left(\theta_{1}, \ldots, \theta_{n}\right)=\left\langle 0\left|\varphi_{i}(0)\right| A_{a_{1}}\left(\theta_{1}\right) \ldots A_{a_{n}}\left(\theta_{n}\right)\right\rangle .
$$

For scalar operators, relativistic invariance requires that the FF only depend on the rapidity differences $\theta_{i}-\theta_{j}$. In integrable quantum field theories the $\mathrm{FF}$ are solutions of some functional

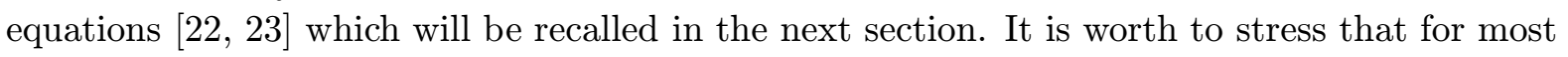
of the operators, it is sufficient to insert into the spectral representations only their one-particle and two-particle $\mathrm{FF}$ in order to reach a very reasonable estimate of the correlators.

In conclusion, both the expansions $\left(\overline{3}-\bar{B}^{\prime}\right)$ and $(\overline{3} . \overline{1} \overline{1})$ converge very rapidly in their domain

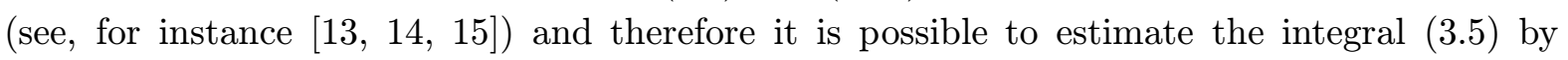
following this procedure:

1. Express the integral in polar coordinates as

$$
\hat{\Gamma}_{j k}^{i}=2 \pi \int_{0}^{+\infty} d r r\left\langle\varphi_{j}(r) \varphi_{k}(0)\right\rangle_{c}^{i}
$$

and split the radial integral into two pieces as

$$
\begin{aligned}
& I=\int_{0}^{+\infty} d r r\left\langle\varphi_{j}(r) \varphi_{k}(0)\right\rangle_{c}^{i} \\
& =\int_{0}^{R} d r r\langle\ldots\rangle_{c}^{i}+\int_{R}^{+\infty} d r r\langle\ldots\rangle_{c}^{i} \\
& \equiv I_{1}(R)+I_{2}(R) .
\end{aligned}
$$

2. Use the best available short-distance representation of the correlator to evaluate $I_{1}(R)$ as well as the best available estimate of its large-distance representation to evaluate $I_{2}(R)$.

3. Optimize the choice of the parameter $R$ in such a way to obtain the best evaluation of the whole integral. In practice, this means looking at that value of $R$ for which a plateau is obtained for the sum of $I_{1}(R)$ and $I_{2}(R)$. Said in another way, $R$ belongs to that interval where there is an overlap between the short-distance and the long-distance expansion of the correlator.

The above methods have been applied to compute universal ratios of several interesting univer-

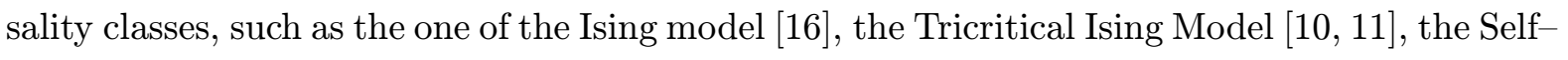

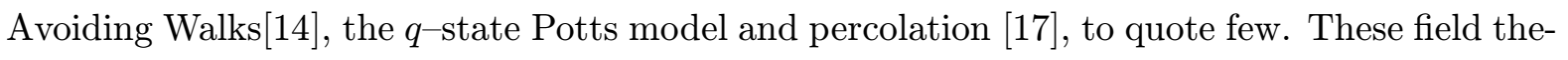

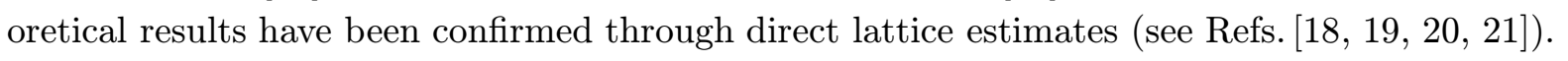




\section{Disorder Operator and Fermion-Boson Correspondence}

From the above discussion, we have seen that a great deal of information can be extracted for the scaling region of a model also in the absence of an exact expression of the correlation functions. It is however an important theoretical problem to see whether it is possible to obtain a closed and exact formula for these quantities. It goes without saying that, in general, this is quite a difficult problem, partially solved only for few models. In the second part of this talk, we shall discuss a particularly interesting example of such a computation, which leads to a differential equation ruling the correlators. Our presentation is based on the results presented

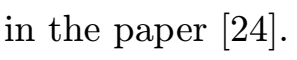

In order to start our discussion, let us consider the (euclidean) action

$$
\mathcal{A}=\int d^{2} x\left[\partial_{\mu} \phi^{*} \partial^{\mu} \phi+m^{2} \phi^{*} \phi+W\left(\phi^{*}, \phi\right)\right]
$$

where the potential $W$ is invariant under the $Z_{N}$ transformation $\phi \rightarrow e^{2 i \pi / N} \phi, \phi^{*} \rightarrow e^{-2 i \pi / N} \phi^{*}$. Let $\left|0_{j}\right\rangle, j=0,1, \ldots, N-1$, be the $N$ vacuua of the broken phase of this theory, with $\mu_{k}(x)$, $k=1, \ldots, N-1$, the disorder operators which create the excitations (kink states) interpolating between the vacua $\left|0_{j}\right\rangle$ and $\left|0_{j+k(\bmod N)}\right\rangle$. These operators carry $k$ units of topological charge and satisfy the conjugation relation $\mu_{k}^{*}(x)=\mu_{N-k}(x)$. The mutual non-locality between the order and disorder operators gives rise to a phase factor when they go around each other in the euclidean plane $\left(z=x_{1}+i x_{2}, \bar{z}=x_{1}-i x_{2}\right)$ :

$$
\begin{aligned}
\phi\left(z e^{2 i \pi}, \bar{z} e^{-2 i \pi}\right) \mu_{k}(0,0) & =e^{\frac{2 i \pi k}{N}} \phi(z, \bar{z}) \mu_{k}(0,0), \\
\phi^{*}\left(z e^{2 i \pi}, \bar{z} e^{-2 i \pi}\right) \mu_{k}(0,0) & =e^{-\frac{2 i \pi k}{N}} \phi^{*}(z, \bar{z}) \mu_{k}(0,0) .
\end{aligned}
$$

These relations are valid along any $Z_{N}$-invariant renormalization group trajectory flowing out of the phase transition point and characterise the operators $\mu_{k}(x)$ beyond their initial role of kink creation operators in the broken phase. The theory $\left(\overline{4}_{1}^{4} \overline{1}_{i}\right)$ with $W=0$ is a particularly simple example of such a trajectory for which, as we will see, the correlation functions of disorder operators remain non-trivial due to the non-locality with respect to the boson.

The correlation functions of non-local operators in two-dimensional free massive theories were extensively studied from the point of view of the isomonodromy theory of differential

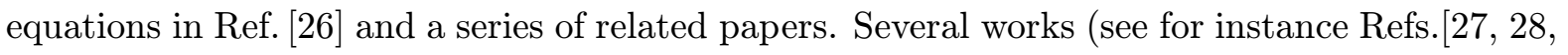
든. general methods of quantum field theory. As we have seen above, an important approach to compute the correlation functions is the one based on the form factors, in which the correlators are expressed as sums over multiparticle asymptotic states. In our example this series can be explicitly resummed and the correlators are given in terms of solutions of non-linear differential equations. Moreover, there is a simple unified treatment of the bosonic and fermionic cases, with the final result expressed by the correspondence ${ }^{1}$

$$
\left\langle\tilde{\mu}_{j}(x) \tilde{\mu}_{k}(0)\right\rangle=\frac{1}{\left\langle\tilde{V}_{j / N}(x) \tilde{V}_{k / N}(0)\right\rangle},
$$

\footnotetext{
${ }^{1}$ We use the notation $\tilde{\Phi}(x) \equiv \Phi(x) /\langle\Phi\rangle$.
} 
where the correlators on the l.h.s. are computed in the theory ( $\left(\overline{4} . \bar{l}_{1}^{1}\right)$ with $W=0$, and those on the r.h.s. refer to the operators $V_{\alpha}(x)=\exp [i \sqrt{4 \pi} \alpha \varphi(x)]$ in the sine-Gordon theory

$$
\mathcal{A}_{s G}=\int d^{2} x\left[\frac{1}{2} \partial_{\nu} \varphi \partial^{\nu} \varphi-\mu \cos \beta \varphi\right]
$$

with $\beta=\sqrt{4 \pi}$ and $\mu$ a suitably chosen mass scale. The theory (4).3it) with $\beta=\sqrt{4 \pi}$ is in fact a free fermionic theory [ij2i] antisolitons interpolating between adjacent vacua of the periodic potential. Their interaction is attractive as long as $\beta<\sqrt{4 \pi}$ and they form topologically neutral bound states, the lightest one being the particle interpolated by the bosonic field in the action ( $\left(\overline{4} \cdot 3^{3}\right)$. These neutral particles are absent from the spectrum of asymptotic states in the repulsive region $\beta>\sqrt{4 \pi}$, and at the point $\beta=\sqrt{4 \pi}$ where the solitons behave as free Dirac fermions.

Since the solitons are non-local in terms of the field $\varphi(x)$, the evaluation of both sides of Eq. (1). respect to non-interacting particles, bosons for the l.h.s. and fermions for the r.h.s. The different statistics of the two particles is responsible for the inversion in Eq. (4.2.

Consider then a theory of free, charge conjugated particles $A$ and $\bar{A}$ with mass $m$, and denote by $\Phi_{\alpha}(x)$ a scalar operator having a non-locality phase $e^{2 i \pi \alpha}\left(e^{-2 i \pi \alpha}\right)$ with respect to (the field which interpolates) the particle $A(\bar{A})$. Acting on the vacuum state $|0\rangle$, such an operator produces neutral states consisting of pairs $A(\theta) \bar{A}(\beta)$. The equations satisfied by the form factors of these theories

$$
\begin{aligned}
& f_{n}^{\alpha}\left(\theta_{1}, \ldots, \theta_{n}, \beta_{1}, \ldots, \beta_{n}\right)= \\
& =\left\langle 0\left|\tilde{\Phi}_{\alpha}(0)\right| A\left(\theta_{1}\right), \ldots, A\left(\theta_{n}\right), \bar{A}\left(\beta_{1}\right), \ldots, \bar{A}\left(\beta_{n}\right)\right\rangle
\end{aligned}
$$

are a particular case of those holding for generic integrable theories (see e.g. [2 $\left.2 \underline{g}_{1}\right]$ )

$$
\begin{aligned}
& f_{n}^{\alpha}\left(\theta_{1}, \ldots, \theta_{i}, \theta_{i+1}, \ldots, \theta_{n}, \beta_{1}, \ldots, \beta_{n}\right)= \\
& =S f_{n}^{\alpha}\left(\theta_{1}, \ldots, \theta_{i+1}, \theta_{i}, \ldots, \theta_{n}, \beta_{1}, \ldots, \beta_{n}\right), \\
& f_{n}^{\alpha}\left(\theta_{1}+2 i \pi, \theta_{2}, \ldots, \theta_{n}, \beta_{1}, \ldots, \beta_{n}\right)= \\
& =S e^{2 i \pi \alpha} f_{n}^{\alpha}\left(\theta_{1}, \ldots, \theta_{n}, \beta_{1}, \ldots, \beta_{n}\right), \\
& \operatorname{Res}_{\theta_{1}-\beta_{1}=i \pi} f_{n}^{\alpha}\left(\theta_{1}, \ldots, \theta_{n}, \beta_{1}, \ldots, \beta_{n}\right)= \\
& =i S^{n-1}\left(1-e^{2 i \pi \alpha}\right) f_{n-1}^{\alpha}\left(\theta_{2}, . ., \theta_{n}, \beta_{2}, . ., \beta_{n}\right),
\end{aligned}
$$

where

$$
S=\left\{\begin{array}{c}
1 \text { for free bosons } \\
-1 \text { for free fermions }
\end{array}\right.
$$

It is easy to check that the solution of the above system of equations is given by

$$
\begin{aligned}
& f_{n}^{\alpha}\left(\theta_{1}, \ldots, \theta_{n}, \beta_{1}, \ldots, \beta_{n}\right)=S^{n(n+2) / 2}(-\sin \pi \alpha)^{n} \\
& \times e^{\left(\alpha-\frac{1}{2} \delta_{S, 1}\right) \sum_{i=1}^{n}\left(\theta_{i}-\beta_{i}\right)}\left|A_{n}\right|_{(S)},
\end{aligned}
$$

where $A_{n}$ is a $n \times n$ matrix $\left(A_{0} \equiv 1\right)$ with entries

$$
A_{i j}=\frac{1}{\cosh \frac{\theta_{i}-\beta_{j}}{2}},
$$


and $\left|A_{n}\right|_{(S)}$ denotes the permanent ${ }^{2}$ of $A_{n}$ for $S=1$ and the determinant of $A_{n}$ for $S=-1$.

The two-point correlators are then given by

$$
\begin{gathered}
G_{\alpha, \alpha^{\prime}}^{(S)}(t)=\left\langle\tilde{\Phi}_{\alpha}(x) \tilde{\Phi}_{\alpha^{\prime}}(0)\right\rangle=\sum_{n=0}^{\infty} \frac{1}{(n !)^{2}(2 \pi)^{2 n}} \\
\int d \theta_{1} \ldots d \theta_{n} d \beta_{1} \ldots d \beta_{n} g_{n}^{\left(\alpha, \alpha^{\prime}\right)}\left(t \mid \theta_{1}, \ldots, \beta_{n}\right),
\end{gathered}
$$

where

$$
\begin{aligned}
& g_{n}^{\left(\alpha, \alpha^{\prime}\right)}(t)=f_{n}^{\alpha}\left(\theta_{1}, \ldots, \beta_{n}\right) f_{n}^{\alpha^{\prime}}\left(\beta_{n}, \ldots, \theta_{1}\right) e^{-t e_{n}} \\
= & \left(S \sin \pi \alpha \sin \pi \alpha^{\prime}\right)^{n} e^{\left(\alpha-\alpha^{\prime}\right) \sum_{i=1}^{n}\left(\theta_{i}-\beta_{i}\right)} \\
& \times\left|A_{n}\right|_{(S)}^{2} e^{-t e_{n}}
\end{aligned}
$$

and

$$
t=m|x| \quad, \quad e_{n}=\sum_{k=1}^{n}\left(\cosh \theta_{k}+\cosh \beta_{k}\right) .
$$

Analogously to the procedure followed in [3inㅁ]

$$
\begin{aligned}
& M_{i j} \equiv M\left(\theta_{i}, \beta_{j}\right)=\left(\sin \pi \alpha \sin \pi \alpha^{\prime}\right)^{1 / 2} e^{-\frac{t}{2} \cosh \theta_{i}} \\
& \times \frac{h\left(\theta_{i}\right) h^{-1}\left(\beta_{j}\right)}{\cosh \frac{\theta_{i}-\beta_{j}}{2}} e^{-\frac{t}{2} \cosh \beta_{j}}
\end{aligned}
$$

where

$$
h(\theta)=e^{\left(\alpha-\alpha^{\prime}\right) \theta / 2}
$$

and rewrite $g^{\left(\alpha, \alpha^{\prime}\right)}$ as

$$
g_{n}^{\left(\alpha, \alpha^{\prime}\right)}=S^{n}\left|M_{n}\right|_{(S)}^{2}=\left|\begin{array}{cc}
0 & M_{n} \\
M_{n}^{T} & 0
\end{array}\right|_{(S)} .
$$

Finally we symmetrise with respect to the two sets of rapidities by introducing a charge index $\varepsilon$ which is 1 for a particle $A$ and -1 for $\bar{A}$, so that we express the correlation functions as

$$
\begin{aligned}
G_{\alpha, \alpha^{\prime}}^{(S)}(t) & =\sum_{L=0}^{\infty} \frac{1}{L !(2 \pi)^{L}} \sum_{\epsilon_{1} \ldots \epsilon_{L}} \int d \theta_{1} \ldots d \theta_{L} \\
& \times\left|K_{\epsilon_{i} \epsilon_{j}}\left(\theta_{i}, \theta_{j}\right)\right|_{(S)}
\end{aligned}
$$

where we have introduced the $L \times L$ matrices with entries

$$
\begin{aligned}
& K_{+-}(\theta, \beta)=M(\theta, \beta), \\
& K_{-+}(\theta, \beta)=M(\beta, \theta), \\
& K_{++}(\theta, \beta)=K_{--}(\theta, \beta)=0 .
\end{aligned}
$$

The last equation in (4.12) ensures that only the terms with $L=2 n$ occur in (4.11). Notice that the dependence on the statistics in (4.11) only reduces to taking the permanent or the

\footnotetext{
${ }^{2}$ The permanent of a matrix differs from the determinant by the omission of the alternating sign factors $(-1)^{i+j}$.
} 
determinant of the same matrix. According to the theory of Fredholm integral operators (see e.g. [i] $\overline{3} \bar{i}])$ the above expression is equal to

$$
G_{\alpha, \alpha^{\prime}}^{(S)}(t)=\operatorname{Det}(1+2 \pi \mathbf{K})^{-S}
$$

so that the correlators of operators having the same non-locality phase in the free fermion and free boson theories are the inverse of each other. This conclusion was first obtained in $[\overline{2} \overline{6} \overline{0}]$ studying the deformation theory of differential equations. The result ( $(\overline{4} . \overline{2} . \bar{j})$ follows from the fact that the operators $V_{\alpha}(x)$ have a non-locality phase $e^{2 i \pi \alpha}$ around the solitons at the sine-Gordon

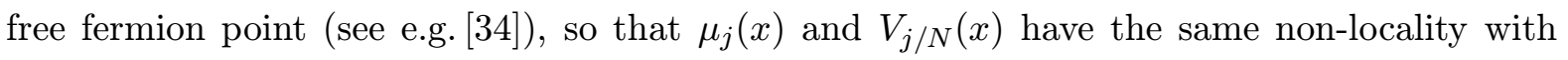
respect to the corresponding particles. Since $j$ runs between 1 and $N-1$, we are actually working with $0<\alpha=j / N<1$.

Once Eq. ( $\left.1 \overline{2} \cdot{ }^{\prime}\right)$ has been obtained by using the large distance expansion of the correlators, it is an interesting check to see how this inversion relation between the two correlators works in the short distance limit. Both in the bosonic and fermionic case the operators $\Phi_{\alpha}(x)$ satisfy the OPE

$$
\left\langle\Phi_{\alpha}(x) \Phi_{\alpha^{\prime}}(0)\right\rangle \sim|x|^{-\Gamma_{\alpha, \alpha^{\prime}}^{(S)}}\left\langle\Phi_{\alpha+\alpha^{\prime}}\right\rangle+\ldots
$$

with

$$
\Gamma_{\alpha, \alpha^{\prime}}^{(S)}=X_{\alpha}^{(S)}+X_{\alpha^{\prime}}^{(S)}-X_{\alpha+\alpha^{\prime}}^{(S)}
$$

$X_{\alpha}^{(S)}$ being the scaling dimensions. In the bosonic case the index $\alpha+\alpha^{\prime}$ is taken modulo 1 . The scaling dimensions can be computed through the formula [1]

$$
X_{\alpha}^{(S)}=-\frac{1}{2 \pi} \int d^{2} x\left\langle\Theta(x) \tilde{\Phi}_{\alpha}(0)\right\rangle_{\text {connected }},
$$

where $\Theta(x)$ is the trace of the energy-momentum tensor. Since the only non-zero form factor of this operator in the free theories is

$$
\langle 0|\Theta(0)| A(\theta) \bar{A}(\beta)\rangle=2 \pi m^{2}\left[-i \sinh \frac{\theta-\beta}{2}\right]^{\delta_{S,-1}},
$$

one easily finds

$$
X_{\alpha}^{(S)}= \begin{cases}\alpha(1-\alpha), & S=1 \\ \alpha^{2}, & S=-1\end{cases}
$$

in agreement with the conjugation properties $\mu_{j}^{*}=\mu_{N-j}$ and $V_{\alpha}^{*}=V_{-\alpha}$. These results coincide

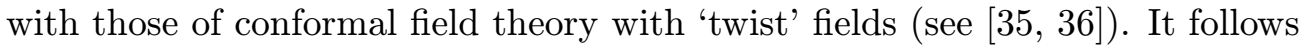

$$
\begin{gathered}
\Gamma_{\alpha, \alpha^{\prime}}^{(-)}=-2 \alpha \alpha^{\prime}, \\
\Gamma_{\alpha, \alpha^{\prime}}^{(+)}= \begin{cases}2 \alpha \alpha^{\prime}, & \alpha+\alpha^{\prime}<1 \\
2\left[\alpha \alpha^{\prime}+1-\left(\alpha+\alpha^{\prime}\right)\right], & \alpha+\alpha^{\prime}>1 .\end{cases}
\end{gathered}
$$

The agreement for $1<\alpha+\alpha^{\prime}<2$ is obtained by observing that in this range of $\alpha+\alpha^{\prime}$, the leading short distance term in the fermionic case is not the one in (4.14) but the first off-critical contribution

$$
\mu / 2 \int d^{2} y\left\langle V_{\alpha}(x) V_{\alpha}(0)\left[V_{1}(y)+V_{-1}(y)\right]\right\rangle_{\mu=0},
$$


which indeed behaves as $|x|^{2\left[\alpha \alpha^{\prime}+1-\left(\alpha+\alpha^{\prime}\right)\right]}$ when $x \rightarrow 0$.

In general, the two-point functions we are dealing with can be expressed as

$$
G_{\alpha, \alpha^{\prime}}^{(S)}(t)=e^{S \Upsilon_{\alpha, \alpha^{\prime}}(t)} .
$$

where $\Upsilon_{\alpha, \alpha^{\prime}}(t)$ is given by $\left[\overline{2} \overline{2} \underline{6}_{1}^{\prime}, \underline{\overline{3}} \overline{1}\right]$

$$
\begin{aligned}
& \Upsilon_{\alpha, \alpha^{\prime}}(t)=\frac{1}{2} \int_{t / 2}^{\infty} \rho d \rho \times \\
& \times\left[\left(\partial_{\rho} \chi\right)^{2}-4 \sinh ^{2} \chi-\frac{\left(\alpha-\alpha^{\prime}\right)^{2}}{\rho^{2}} \tanh \chi\right],
\end{aligned}
$$

with $\chi(\rho)$ satisfying the differential equation

$$
\partial_{\rho}^{2} \chi+\frac{1}{\rho} \partial_{\rho} \chi=2 \sinh 2 \chi+\frac{\left(\alpha-\alpha^{\prime}\right)^{2}}{\rho^{2}} \tanh \chi\left(1-\tanh ^{2} \chi\right)
$$

with suitable boundary conditions. The short distance behaviour for $\alpha+\alpha^{\prime}<1$ is given by

$$
\lim _{t \rightarrow 0} G_{\alpha, \alpha^{\prime}}^{(S)}(t)=\left(C_{\alpha, \alpha^{\prime}} t^{2 \alpha \alpha^{\prime}}\right)^{-S}
$$

with an amplitude that can be deduced from the work of Ref. [i] on vacuum expectation values in the sine-Gordon model:

$$
\begin{aligned}
& C_{\alpha, \alpha^{\prime}}=2^{-2 \alpha \alpha^{\prime}} \times \\
& \exp \left\{2 \int _ { 0 } ^ { \infty } \frac { d t } { t } \left[\frac{\sinh \alpha t \cosh \left(\alpha+\alpha^{\prime}\right) t \sinh \alpha^{\prime} t}{\sinh ^{2} t}+\right.\right. \\
& \left.\left.-\alpha \alpha^{\prime} e^{-2 t}\right]\right\} .
\end{aligned}
$$

Finally, let us discuss the case in which we have a $Z_{2}$ symmetry. This is somehow special since a broken phase with two degenerate vacua can be realised in terms of a neutral boson. In fact, a disorder operator with non-locality factor -1 is present also in the theory of a neutral bosonic free particle. It is easy to check that, for $S=1$ and $\alpha=1 / 2$, the form factors (4. imply the factorisation

$$
\mu_{1}=\mu_{(1)} \times \mu_{(2)}, \quad N=2
$$

where $\mu_{(j)}$ is the disorder operator with scaling dimension $X_{1 / 2}^{(+)} / 2=1 / 8$ associated to the neutral boson $A_{j}$ entering the decomposition $A=\left(A_{1}+i A_{2}\right) / \sqrt{2}, \bar{A}=\left(A_{1}-i A_{2}\right) / \sqrt{2}$.

The fermion-boson correspondence observed above for charged particles has an analogue in the neutral case, and the correlation function

$$
G(t)=\left\langle\tilde{\mu}_{(j)}(x) \tilde{\mu}_{(j)}(0)\right\rangle=\left[G_{1 / 2,1 / 2}^{(+)}(t)\right]^{1 / 2}
$$

can be related to correlators computed in the theory of a free neutral fermion, i.e. in the Ising field theory without magnetic field. To see this, let us recall that the correlators of the spin and disorder operators in the unbroken phase of the scaling Ising model can be written as [205]

$$
\begin{aligned}
& \tau_{ \pm}(t) \equiv\langle\tilde{\mu}(x) \tilde{\mu}(0)\rangle \pm\langle\sigma(x) \sigma(0)\rangle= \\
& =\exp \left[ \pm \frac{1}{2} \chi(t / 2)-\frac{1}{2} \Upsilon_{1 / 2,1 / 2}(t)\right]
\end{aligned}
$$




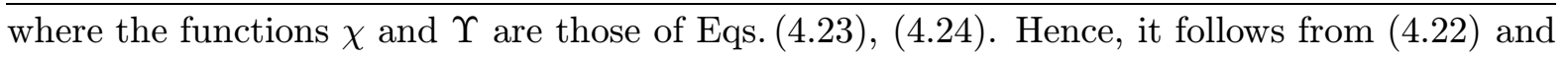
$(4 . \overline{2} \overline{8})$ that

$$
G(t)=\left[\tau_{+}(t) \tau_{-}(t)\right]^{-1 / 2} .
$$

Concerning the short distance behaviour of this correlator, the power law (4.25i) acquires in this case a logarithmic correction due to the 'resonance' with the leading off-critical contribution $\left(4 . \overline{2} \overline{1}_{-1}\right)$. Such a contribution to the correlator $\left\langle\tilde{V}_{\alpha}(x) \tilde{V}_{\alpha}(0)\right\rangle$ behaves as $-C_{\alpha, \alpha} t^{1 / 2}[1+(2-4 \alpha) \ln t]$ in the limit $\alpha \rightarrow 1 / 2$, so that

$$
\lim _{t \rightarrow 0} G_{1 / 2,1 / 2}^{(-)}(t)=\lim _{t \rightarrow 0} G^{-2}(t)=\mathcal{B} t^{1 / 2} \ln (1 / t),
$$

with

$$
\mathcal{B}=-4 \operatorname{Res}_{\alpha=1 / 2} C_{\alpha, \alpha}=0.588353 . .
$$

and this amplitude coincides with that of the product $\tau_{+}(t) \tau_{-}(t)$ in the Ising model.

The considerations of this section have recently been extended to the case of massive ghost theories [i] $\left.{ }_{3}^{3} \overline{7}\right]$. In all these cases the correlation functions of disorder operators can be computed exactly for free theories and are related to the same differential equation (4.24). In absence of interaction, the correlators are non-trivial due to the non-locality between the operators and the particles. The resummation of the spectral series for interacting theories remains a challanging task even in presence of integrability.

\section{Non-integrable Deformations}

The free bosonic and fermionic theories discussed above can also be regarded as describing phases of spontaneously broken $Z_{N}$ symmetry. In this dual vision, the excitations are free kinks $\left|K_{j, j \pm 1}(\theta)\right\rangle$ interpolating between two adjacent vacua $\left|0_{j}\right\rangle$ and $\left|0_{j \pm 1}\right\rangle$ (the indices are taken modulo $N$ ). Let us denote by $\Phi_{k / N}, k=0,1, \ldots, N-1$, the operators we are interested in. They correspond to the exponential operators $V_{k / N}$ in the fermionic case ${ }^{3}$, and to the operators $\sigma_{k}$, dual to the disorder operators $\mu_{k}$, in the bosonic case. These operators create multikink excitations with zero topologic charge, i.e. starting and ending in the same vacuum state. Form factors on kink states were discussed in [i] $\left.\overline{7}_{1}\right]$. Consider for the sake of simplicity the two-kink matrix elements

$$
F_{j, k}^{ \pm}\left(\theta_{1}-\theta_{2}\right) \equiv\left\langle 0_{j}\left|\Phi_{k / N}(0)\right| K_{j, j \pm 1}\left(\theta_{1}\right) K_{j \pm 1, j}\left(\theta_{2}\right)\right\rangle,
$$

satisfying the equations

$$
\begin{aligned}
F_{j, k}^{ \pm}(\theta)= & S F_{j, k}^{\mp}(-\theta) \\
F_{j, k}^{ \pm}(\theta+2 i \pi)= & F_{j \pm 1, k}^{\mp}(-\theta) \\
\operatorname{Res}_{\theta=i \pi} F_{j, k}^{ \pm}(\theta)= & i\left[\left\langle 0_{j}\left|\Phi_{k / N}\right| 0_{j}\right\rangle+\right. \\
& \left.-\left\langle 0_{j \pm 1}\left|\Phi_{k / N}\right| 0_{j \pm 1}\right\rangle\right] .
\end{aligned}
$$

\footnotetext{
${ }^{3}$ In this case the $\mathrm{N}$ degenerate vacua can be identified with those of the periodic potential in $\left(\overline{4}-\overline{4} .3_{1}^{\prime}\right)$ identified modulo N.
} 
Since the generator $\Omega$ of $Z_{N}$ transformations $\left(\Omega^{N}=1\right)$ acts on states and operators as $(\omega \equiv$ $\left.e^{2 i \pi / N}\right)$

$$
\begin{aligned}
& \Omega\left|K_{j, j+1}\left(\theta_{1}\right) K_{j+1, j+2}\left(\theta_{2}\right) \ldots\right\rangle= \\
& =\left|K_{j+1, j+2}\left(\theta_{1}\right) K_{j+2, j+3}\left(\theta_{2}\right) \ldots\right\rangle, \\
& \Omega^{-1} \Phi_{k / N}(x) \Omega=\omega^{k} \Phi_{k / N}(x),
\end{aligned}
$$

the above form factor equations can be rewritten as

$$
\begin{aligned}
F_{j, k}^{ \pm}(\theta+2 i \pi) & =S \omega^{ \pm k} F_{j, k}^{ \pm}(\theta), \\
\operatorname{Res}_{\theta=i \pi} F_{j, k}^{ \pm}(\theta) & =i\left(1-\omega^{ \pm k}\right)\left\langle 0_{j}\left|\Phi_{k / N}\right| 0_{j}\right\rangle .
\end{aligned}
$$

Once the identifications $A \longleftrightarrow K_{i, i+1}, \bar{A} \longleftrightarrow K_{i, i-1}$ are made, these relations are equivalent to the previous equations for the Form Factors with $n=1$. This correspondence is easily extended to all values of $n$ and leads to the same form factors and correlation functions discussed for the unbroken phase.

The introduction of a 'magnetic field' pointing in the $k$ direction and breaking explicitely the $Z_{N}$ symmetry corresponds to adding to the free action a term

$$
h \int d^{2} x \Psi_{k}(x)
$$

where

$$
\Psi_{k}(x)=\frac{1}{N} \sum_{l=0}^{N-1} \omega^{-k l} \Phi_{l / N}(x) .
$$

The first order corrections to the energy density $\varepsilon_{j}$ of the vacuum state $\left|0_{j}\right\rangle$ and to the mass $m_{j, j \pm 1}$ of the kink $K_{j, j \pm 1}$ can be computed according to the Form Factor Perturbation Theory

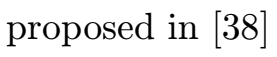

$$
\begin{aligned}
& \delta \varepsilon_{j} \sim h\left\langle 0_{j}\left|\Psi_{k}\right| 0_{j}\right\rangle=h v \delta_{j, k}, \\
& \delta m_{j, j \pm 1}^{2} \sim h\left\langle 0_{j}\left|\Psi_{k}(0)\right| K_{j, j \pm 1}(i \pi) K_{j \pm 1, j}(0)\right\rangle .
\end{aligned}
$$

It follows from the residue equation written above that

$$
\begin{aligned}
& \operatorname{Res}_{\theta=i \pi}\left\langle 0_{j}\left|\Psi_{k}(0)\right| K_{j, j \pm 1}(\theta) K_{j \pm 1, j}(0)\right\rangle= \\
& \frac{i v}{N}\left(\delta_{j, k}-\delta_{j, k \mp 1}\right),
\end{aligned}
$$

This formula implies that the correction to the mass of the kinks interpolating between the vacua $\left|0_{k}\right\rangle$ and $\left|0_{k \pm 1}\right\rangle$ is infinite. This divergence simply reflects the fact that these kinks become unstable because the magnetic field removes the degeneracy of the vacuum $\left|0_{k}\right\rangle$ with the two adjacent vacua. These kinks are then confined in pairs $K_{k, k \pm 1} K_{k \pm 1, k}$ and the confinement gives rise to a string of bound states with zero topologic charge, as discussed in more details in [i] $\left.{ }^{3} \overline{9}\right]$.

\section{Acknowledgments}

This work was supported by the TMR Network "EUCLID. Integrable models and applications: from strings to condensed matter", contract number HPRN-CT-2002-00325. 


\section{References}

[1] V. Privman, P.C. Hohenberg and A. Aharony, Universal Critical-Point Amplitude Relations, in Phase Transitions and Critical Phenomena, edited by C. Domb and J.L. Lebowitz (Academic Press Limited, London, 1991), Vol. 14.

[2] A.A. Belavin, A.M. Polyakov and A.B. Zamolodchikov, Nucl. Phys. B 241 (1994), 333.

[3] D. Friedan, Z. Qiu, S. Shenker, Phys. Rev. Lett. 52 (1984), 1575

[4] Al.B. Zamolodchikov, Nucl. Phys. B 342 (1990), 695.

[5] V.A. Fateev, Phys. Lett. B 324 (1994), 45.

[6] V.P. Yurov and Al.B. Zamolodchikov, Int. J. Mod. Phys. A 6 (1991), 4557.

[7] S.Lukyanov and A.B. Zamolodchikov, Nucl. Phys. B 493 (1997), 571.

[8] V. Fateev, S. Lukyanov, A.B. Zamolodchikov, Al.B. Zamolodchikov, Nucl. Phys. B 516 (1998), 652.

[9] R. Guida and N. Magnoli, Phys. Lett. B 411 (1997) 127.

[10] D. Fioravanti, G. Mussardo and P. Simon, Phys. Rev. Lett. 85 (2000), 126.

[11] D. Fioravanti, G. Mussardo and P. Simon, Phys. Rev. E 63 (2001), 16103.

[12] G. Delfino, P. Simonetti and J.L. Cardy, Phys. Lett. B 387 (1996), 327.

[13] Al.B. Zamolodchikov, Nucl. Phys. B 348 (1991), 619.

[14] J.L. Cardy and G. Mussardo, Nucl. Phys. B 410 (1993), 451.

[15] G. Delfino and G. Mussardo, Nucl. Phys. B 455 (1995), 724.

[16] G. Delfino, Phys. Lett. B 419 (1998), 291; Err. ibid. B 518 (2001) 330.

[17] G. Delfino and J.L. Cardy, Nucl. Phys. B 519 (1998), 551.

G. Delfino, G.T. Barkema and J.L. Cardy, Nucl. Phys. B 565 (2000) 521.

G. Delfino and J.L. Cardy, Phys. Lett. B 483 (2000) 303.

[18] M. Caselle, R. Tateo and S. Vinti, Nucl. Phys. B 562 (1999) 549.

[19] M. Caselle and M. Hasenbusch, Nucl. Phys. B 579 (2000) 667.

[20] L. Shchur, P. Butera, B. Berche, Nucl. Phys. B 620 (2002) 579.

[21] I.G. Enting and A.J. Guttmann, Susceptibility amplitudes for the 3- and 4-state Potts models, Physica A, in press.

[22] M. Karowski, P. Weisz, Nucl. Phys. B 139 (1978), 445.

[23] F.A. Smirnov, Form Factors in Completely Integrable Models of Quantum Field Theory (World Scientific), 1992 and references therein.

[24] G. Delfino, P. Grinza and G. Mussardo, Phys. Lett. B 536 (2002), 169.

[25] T.T. Wu, B.M. McCoy, C.A. Tracy and E. Barouch, Phys. Rev. B 13 (1976) 316.

[26] M. Sato, T. Miwa and M. Jimbo, Publ. RIMS, Kyoto Univ., 15 (1979) 871.

[27] B. Berg, M. Karowski and P. Weisz, Phys. Rev. D 19 (1979), 2477.

[28] E.C. Marino, B. Schroer and J.A. Swieca, Nucl. Phys. B 200 (1982), 473. 
[29] V.P. Yurov and Al.B. Zamolodchikov, Int. J. Mod. Phys. A6 (1991), 3419.

[30] O. Babelon and D. Bernard, Phys. Lett. B288 (1992), 113.

[31] D. Bernard and A. LeClair, Nucl. Phys. B 426 (1994) 534; Erratum-ibid. B 498 (1994) 619;

[32] S. Coleman, Phys. Rev. D 11 (1975), 2088.

[33] J. Schwinger, Phys. Rev. 92 (1953), 1283.

[34] G. Delfino, J. Phys. A 34 (2001) L311.

[35] L. Dixon, D. Friedan, E. Martinec and S. Shenker, Nucl. Phys. B 282 (1987), 13.

[36] Al.B. Zamolodchikov, Nucl. Phys. B 285 (1987), 481.

[37] G. Delfino, P. Mosconi and G. Mussardo, J. Phys. A 36 (2003) L1.

[38] G. Delfino, G. Mussardo and P. Simonetti, Nucl. Phys. B 473 (1996), 469.

[39] G. Delfino and G. Mussardo, Nucl. Phys. B 516 (1998), 675. 